\title{
Doença ateromatosa multivascular: relato de caso
}

\author{
Multivessel atherosclerotic disease: case report
}

\author{
Gustavo Henrique Reis de Oliveira', Antônio Fernando Diniz Freire', \\ Alaís Virgínia Ferreira de Souza', Tânia Maria Gonsalvez Quintão Santana²
}

Oliveira GHR, Freire AFD, Souza AVF, Santana TMGQ. Doença ateromatosa multivascular: relato de caso/Multivessel atherosclerotic disease: case report. Rev Med (São Paulo). 2011 jul.-set.;90(3):144-8.

RESUMO: Objetivos: O envelhecimento da população representa um desafio para o sistema de saúde pelo aumento da incidência de doenças crônico-degenerativas, o que leva a crescentes custos econômicos e sociais. A mortalidade por doenças cardiovasculares aumenta com a idade: a partir dos 50 anos passa a ser a maior e a principal causa de óbitos no Brasil. A aterosclerose é uma dessas doenças, tendo diferentes fatores de riscos. Este relato de caso mostra uma doença multissistêmica grave, e visa mostrar a relação direta dos fatores de risco do estudo de Framingham na gênese da doença aterosclerótica arterial sistêmica. Os conhecimentos gerados poderiam facilitar o planejamento de ações para uma melhor avaliação de pacientes com essas morbidades, assim como a implantação de programas e estratégias que contribuam para melhorar o atendimento e a qualidade de vida de pacientes nesta faixa etária. Relato de Caso: Paciente do sexo masculino, 72 anos, hipertenso, diabético e dislipidêmico, internado no serviço de cardiologia do Hospital Ibiapaba em Barbacena, com quadro de dor precordial e claudicação intermitente. Foi submetido a estudo cineangiocoronariográfico que evidenciou ateromatose coronariana significativa com obstrução de $90 \%$ em coronária direita, $50 \%$ da circunflexa, e $40 \%$ na descendente anterior, além de extensa dilatação aneurismática do ventrículo esquerdo e acinesia de parede inferior e apical. No estudo angiográfico das artérias de membros inferiores foi constatado oclusão de $70 \%$ das ilíacas, entre outros vasos. Conclusões: Atualmente, existe consenso entre os pesquisadores de que são necessárias mais pesquisas sobre as implicações dos fatores de risco para doenças cardiovasculares em idosos, devido a sua importância e impacto significativos. A doença neste segmento da população não é uma consequência inevitável do envelhecimento biológico, mas um processo patológico com fatores de risco que podem ser evitados.

DESCRITORES: Doenças cardiovasculares/diagnóstico; Fatores de risco; Placa aterosclerótica; Estudos transversais; Idoso.
ABSTRACT: Objectives: The rapidly aging population represents a major challenge for the health system, because of the higher incidence of chronic degenerative diseases, which leads to increasing economic and social costs. Mortality from cardiovascular disease is greatly increased with the passage of age, and from fifty years become the largest and the leading cause of deaths in Brazil. Atherosclerosis is one of these diseases, with different risk factors. This article, from a case report of a severe multisystem disease, aims to show the direct relation of risk factors in the Framingham study in the genesis of atherosclerotic arterial disease. The knowledge generated could facilitate the planning of actions to better access patients with these illnesses as well as to develop programs and strategies that contribute to benefit the care and quality of life in this age group. Case Report. A male patient, 72 years, hypertensive, diabetic and dyslipidemic internship at the hospital's cardiology service Ibiapaba in Barbacena with chest pain and intermittent claudication. He underwent coronary angiographic study showed that significant atheromatous coronary obstruction $90 \%$ in right coronary artery, the circumflex $50 \%$ and $40 \%$ left anterior descending, and extensive aneurysmal dilatation of the left ventricle and akinesis of inferior wall and apical. In the angiographic study of arteries of lower limbs was found $70 \%$ occlusion of iliac arteries, among other vessels. Conclusions: There is now consensus among researchers that more research is needed on the implications of risk factors for cardiovascular disease in the elderly because of their importance and impact. The disease in this population segment is not an inevitable consequence of biological aging, but a pathological process with risk factors can be avoided.

KEYWORDS: Cardiovascular disease/diagnosis; Risk factors; Plaque, atherosclerotic; Cross-sectional studies; Aged.

Trabalho apresentado no XVII Congresso da Sociedade Brasileira de Hipertensão e XXIII Sessão Científica da Sociedade Interamericana de Hipertensão, Belo Horizonte, MG, 2009.

1. Acadêmicos de Medicina da Faculdade de Medicina de Barbacena, FUNJOG, Barbacena, MG.

2. Cardiologista, Orientadora deste trabalho, Faculdade de Medicina de Barbacena - FUNJOB, Barbacena, MG.

Endereço para correspondência: Gustavo Henrique Reis de Oliveira. Rua Zacarias El-Corab, 27 - Vila Marchetti, São João Del Rei, MG, Brasil. E-mail: gustavoreisoliveira@yahoo.com.br 


\section{INTRODUÇÃO}

$\mathrm{O}$ rápido processo de envelhecimento populacional brasileiro representa um grande desafio para o sistema de saúde, devido à maior incidência de doenças crônicas e incapacidades físicas, que levam a crescentes custos econômicos e sociais ${ }^{1}$. A mortalidade por doenças cardiovasculares aumenta com o decorrer da idade, sendo que a partir de cinquenta anos passa a ser a maior e a principal causa de óbitos no Brasil.

$A$ aterosclerose é uma doença degenerativa de etiologia multifatorial. Diferentes fatores de risco (genéticos e adquiridos) atuando em conjunto podem determinar sua ocorrência em mais de $50 \%$ da população adulta mundial ${ }^{2}$. Os fatores de risco para aterosclerose podem ser divididos em modificáveis (tabagismo, sedentarismo, obesidade, estresse, hiperlipidemia, hipertensão arterial) e não modificáveis (diabetes mellitus, hipertensão familiar, trombofilias, sexo, idade e hereditariedade) ${ }^{3}$. Indivíduos hipertensos freqüentemente apresentam altos níveis de colesterol, obesidade, freqüência cardíaca elevada, hipertrigliceridemia e diabetes mellitus ${ }^{4}$.

O presente artigo, a partir de um relato de caso de uma doença multissistêmica grave, visa mostrar a relação direta dos fatores de risco do estudo de Framingham na gênese da doença aterosclerótica arterial sistêmica.
Os conhecimentos gerados poderiam facilitar o planejamento de ações para uma melhor avaliação de pacientes com essas morbidades, assim como na implantação de programas e estratégias que contribuam para beneficiar o atendimento e a qualidade de vida de pacientes nesta faixa etária.

\section{RELATO DE CASO}

Paciente do sexo masculino, 72 anos, leucodérmico, hipertenso, diabético e dislipidêmico. Foi internado no serviço de cardiologia do Hospital Ibiapaba de Barbacena com quadro de dor precordial aos esforços e claudicação intermitente. O paciente evolui com incapacidade de deambulação e dor em MMII em repouso.

Em primeira consulta, ao exame, o paciente apresentou-se consciente, orientado, acianótico, anictérico e desidratado, afebril, $\mathrm{PA}=160 \times 100 \mathrm{mmHg}$ e freqüência cardíaca de 110 batimentos por minuto. Aparelho cardiovascular apresentava desvio de Ictus para esquerda, ritmo cardíaco regular em dois tempos, sopro sistólico regurgitativo. Os pulsos estavam diminuídos em membros inferiores.

Foram realizados exames de sangue, que evidenciaram: Glicemia de $314 \mathrm{mg} / \mathrm{dL}$, Triglicérides de $208 \mathrm{mg} / \mathrm{dL}$, colesterol de $215 \mathrm{mg} / \mathrm{dL}$; VLDL de $42 \mathrm{mg} /$ dL. O estudo eletrocardiográfico evidenciou: Taquicardia supraventricular, sobrecarga de ventrículo esquerdo, isquemia de parede inferior evidenciada pela inversão de onda T, e leve infra de ST (Figura 1).

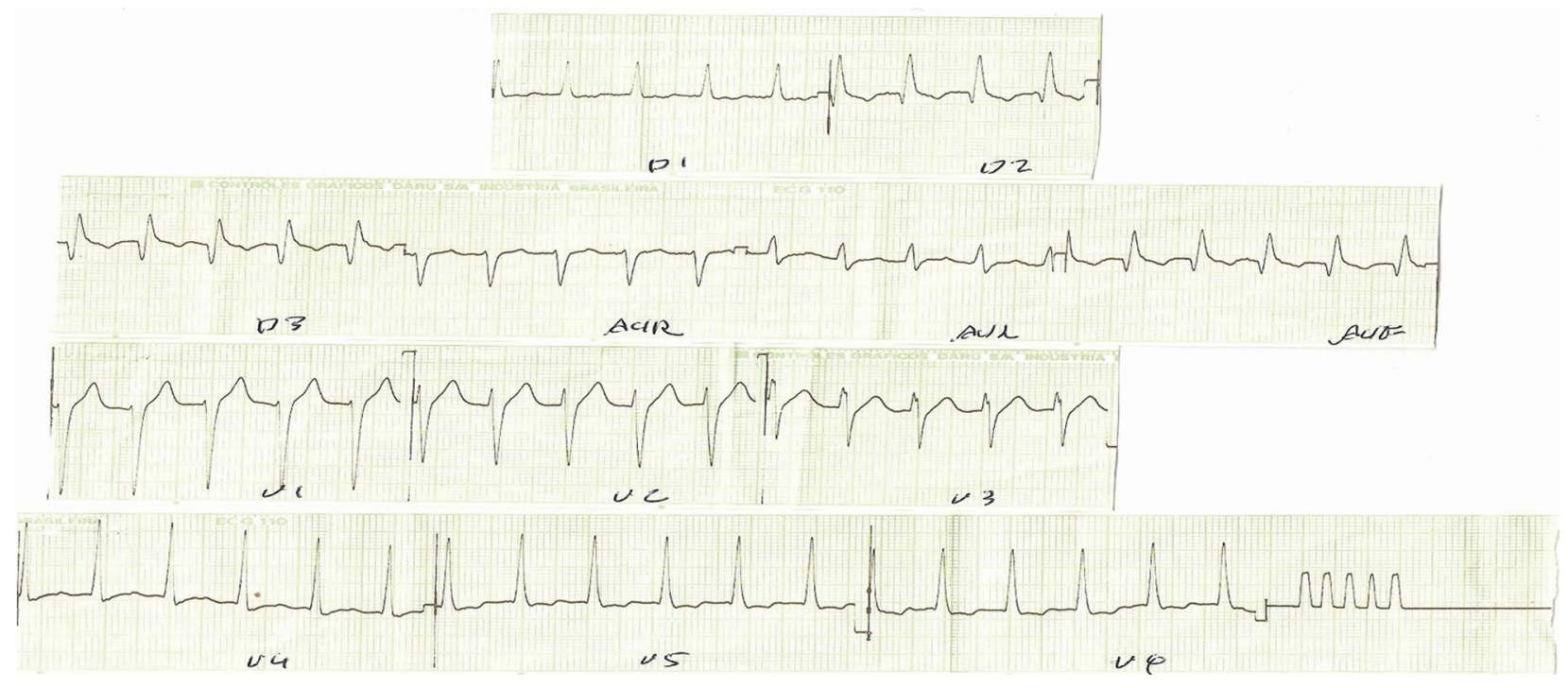

Figura 1. Eletrocardiograma 
O paciente então foi submetido a estudo cineangiocoronariográfico, além de aortografia e cateterismo seletivo das artérias ilíacas comuns direita e esquerda, com seguimento das árvores arteriais dos membros inferiores. O estudo cineangiocoronariográfico evidenciou coronária direita ocluída, tronco da coronária esquerda com obstrução de $30 \%$, descendente anterior com obstruções de até $50 \%$, primeiro ramo diagonal com obstruções de até $40 \%$, artérias diagonais com obstrução de $20 \%$, circunflexa com obstrução de $40 \%$,

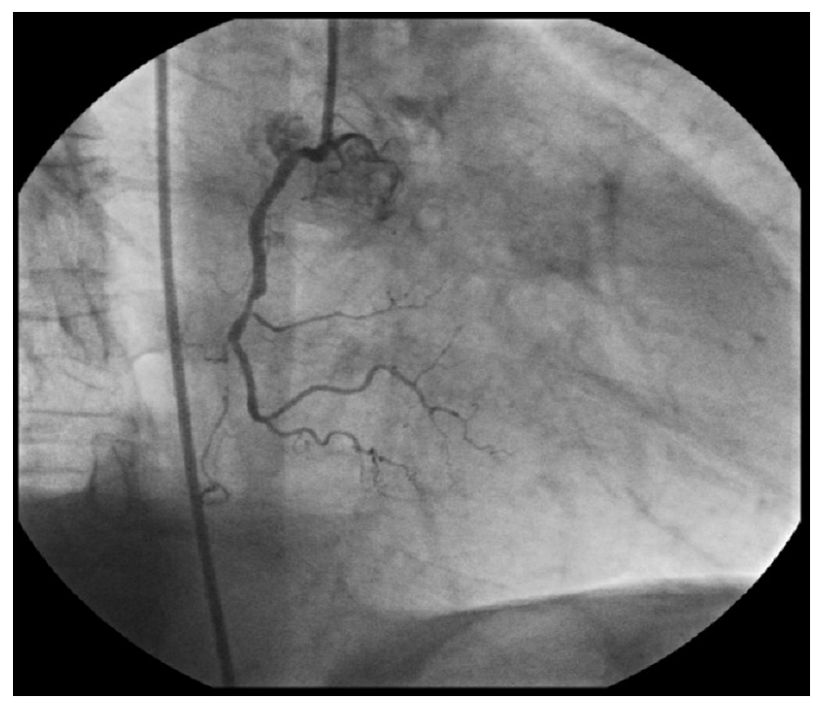

Figura 2. Coronariografia

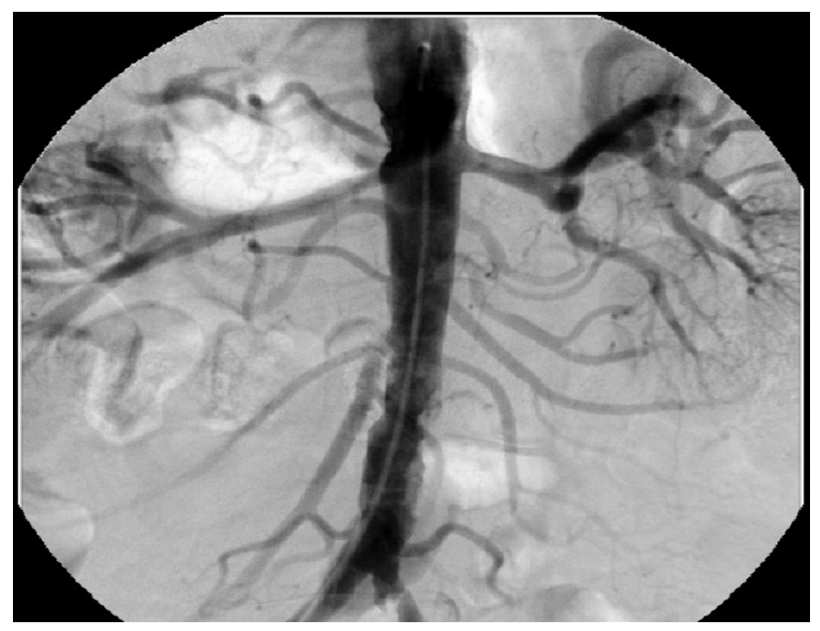

Figura 4. Obstrução de aorta abdominal e artéria renal esquerda

Foi realizada a cirurgia de by-pass. Em um segundo tempo será realizado a intervenção através de angioplastia com stent em coronária direita. Paciente vem evoluindo satisfatoriamente até o momento. segundo ramo marginal esquerdo com obstrução de $60 \%$, ventrículo esquerdo com aneurisma inferior e hipocinesia moderada das demais paredes (Figuras 2 e 3). A aortografia revelou aorta abdominal com obstruções de até $50 \%$ e artéria renal direita com estenose de 40\% (Figura 4). O cateterismo de MMII demonstrou artéria femoral superficial direita ocluída, recanalizando em artéria poplítea e oclusão da artéria fibular esquerda. Foi constatado oclusão de $70 \%$ das ilíacas (Figura 5). Foram evidenciadas também outras obstruções menores.

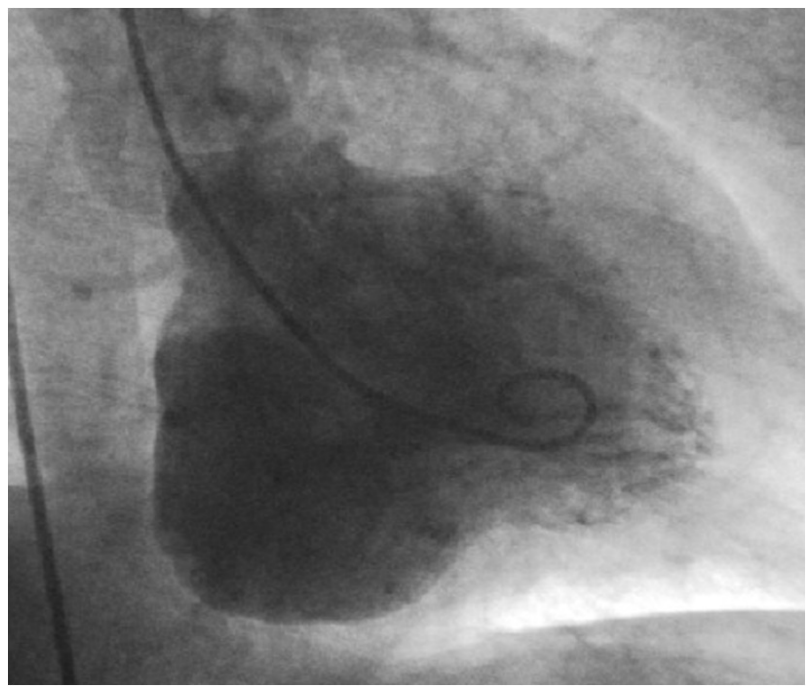

Figura 3. Ventrículo esquerdo com aneurisma inferior

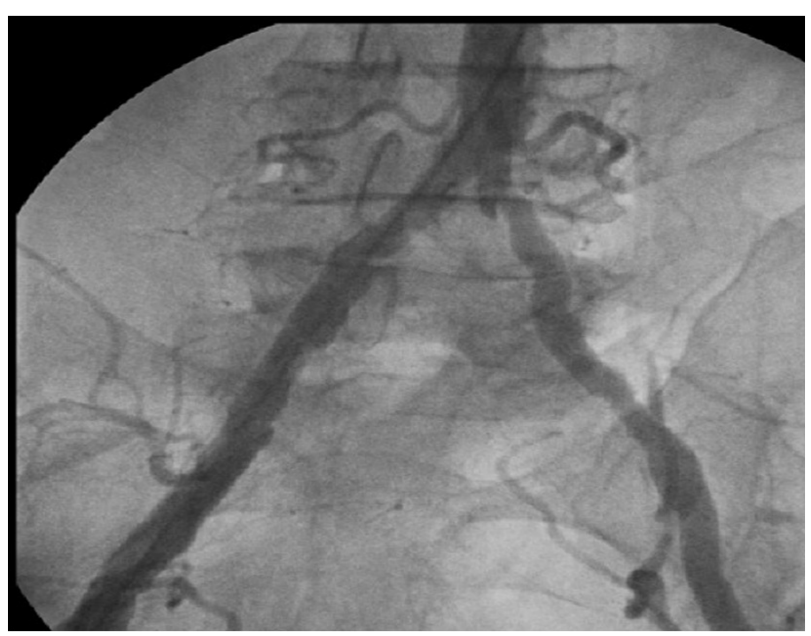

Figura 5. Obstrução de artérias ilíacas

\section{DISCUSSÃo}

Trata-se de um paciente com varias doenças sistêmicas não controladas, que após bateria de exames investigatórios foram evidenciadas várias 
lesões ateromatosas, comprovando a clínica. A gênese da doença ateromatosa multissistêmica é muito bem explicada na literatura pelas co-morbidades do paciente.

Uma série de fatores de risco modificáveis está associada à doença arterial coronária, que também contribui para aterosclerose da circulação extracardíaca. Neste particular, além do tabagismo, da dislipidemia e da hipertensão, o diabetes mellitus tem papel relevante, em especial ao considerar sua crescente incidência, ano a ano, decorrente do envelhecimento das populações e do aumento da obesidade e do sedentarismo. A aterosclerose cardiovascular no diabético é mais extensa e mais grave, com maior propensão à calcificação e maior acometimento distal. No estudo de Framinghan, o diabetes contribui mais como fator de risco para doença arterial periférica do que para doença coronária ou acidente vascular cerebral ${ }^{5}$.

Existem evidências da correlação entre o perfil lipídico e a pressão arterial sistêmica, como observado na síndrome metabólica. Esta contempla a presença invariável de hipertensão arterial sistêmica associada à adiposidade abdominal, à hipertrigliceridemia, ao HDL-C baixo e à glicemia de jejum alterada. Esses achados se traduzem em risco maior de evolução para o diabetes mellitus e para doença cardiovascular. Estudos observacionais demonstraram associação positiva entre níveis de colesterol total e pressão arterial na população em geral e em pacientes hipertensos. A relação causal entre esses dois fatores de risco ainda permanece não elucidada. Sabe-se que os mecanismos fisiopatológicos envolvidos na gênese da hipertensão e da dislipidemia compartilham anormalidades metabólicas comuns, que podem agir sinergicamente ou até mesmo acelerar processo de aterogênese. A hipercolesterolemia pode ter efeito primário nos vasos e no tônus vascular, além de promover a disfunção endotelial, também presente de forma incipiente na hipertensão arterial ${ }^{4}$.

A hipertensão arterial sistêmica acomete aproximadamente $25 \%$ da população mundial, com previsão de aumento de $60 \%$ dos casos da doença em $2025^{6}$. Entre os fatores de risco para mortalidade, a hipertensão arterial sistêmica explica $40 \%$ das mortes por AVC e $25 \%$ por doença coronariana ${ }^{6}$. O estudo de Framingham demonstra que a pressão sistólica aumenta continuamente com a idade em todos os grupos. Dados de 30 anos de seguimento do estudo Framingham mostraram claramente 0 aumento do risco de eventos cardiovasculares com a elevação da pressão sistólica, particularmente no idoso ${ }^{7}$.

De acordo com o Estudo Multicêntrico sobre a
Prevalência de Diabetes no Brasil, realizado em 1988, $7,6 \%$ da população adulta do país tem a doença. E, em 2001, na Campanha Nacional de Detecção de Casos Suspeitos de Diabetes no Brasil, quando foram realizadas 20,7 milhões de glicemia capilar, detectouse prevalência de $14,6 \%$ de exames suspeitos. Além disso, as estatísticas oficiais de morbimortalidade apontam que o diabetes mellitus tipo 2 constitui a quinta indicação de hospitalização no Brasil e está entre as dez maiores causas de mortalidade no país ${ }^{8}$.

O diabetes mellitus tipo 2 é um dos mais graves problemas de saúde pública em todo o mundo, particularmente no Brasil, pela alta prevalência e por se destacar como importante fator de risco cardiovascular'.

Diversos fatores de risco cardiovasculares podem afetar as propriedades funcionais e estruturais das grandes artérias e conseqüentemente promover aumento da rigidez arterial, aumento da espessura da parede da artéria, entre outras modificações. Estudos caso controle conduzidos em várias populações específicas têm demonstrado a influência deletéria do envelhecimento e outros fatores de risco como a hipertensão arterial e o diabetes mellitus, sobre as propriedades funcionais e estruturais das grandes artérias ${ }^{9}$.

Alterações funcionais e estruturais das grandes artérias exercem um importante papel na patogênese das doenças cardiovasculares. $O$ diabetes mellitus pode induzir essas alterações em diferentes territórios arteriais e assim levar ao desenvolvimento de aterosclerose e suas conseqüências cardiovasculares. Tanto o diabetes tipo 1 quanto o tipo 2 são associados com excesso de doença cardiovascular, onde as principais causas de mortalidade são conseqüência de lesões ateroscleróticas de grandes artérias como o infarto do miocárdio e o acidente vascular cerebral. Diversos estudos têm demonstrado alterações funcionais e estruturais de grandes artérias em diabéticos, principalmente aumento da rigidez arterial em diabéticos tanto do tipo 1 quanto do tipo $2^{9}$.

Os mecanismos que levam a aceleração da aterosclerose em diabéticos ainda não são completamente conhecidos. Mas sabe-se que a ação da hiperglicemia sobre os vasos sanguíneos, a resistência insulínica e a associação do diabetes com outros fatores de risco podem favorecer essa condição. Indivíduos com esta enfermidade possuem comumente, uma dislipidemia aterogênica, caracterizada por valores elevados de triglicérides e baixos de HDL - colesterol, assim como de LDL colesterol' ${ }^{1}$.

O papel das dislipidemias como um dos 
mais importantes fatores que contribuem para a aterosclerose está bem documentada na literatura ${ }^{1}$. A dislipidemia é um distúrbio do metabolismo que cursa com a alteração de uma ou mais frações dos lípides séricos, quando então alcançam níveis associados com um aumento de risco cardiovascular ${ }^{10}$. Desde a disfunção endotelial até a ruptura de lesões na parede vascular, pode-se observar a participação ativa da LDL como um agente pró-aterogênico.

Em relação às alterações no perfil lipídico, o impacto desfavorável das dislipidemias sobre a morbimortalidade cardiovascular é amplamente reconhecido, bem como a freqüente associação dessas condições com o diabetes, ocorrendo significativa elevação da mortalidade em indivíduos com diabetes mellitus tipo 1 e 2 na presença de dislipidemias ${ }^{8}$.

\section{REFERÊNCIAS}

1. Silva RCP, Simões MJS, Leite AA. Fatores de risco para doenças cardiovasculares em idosos com diabetes mellitus tipo 2. Rev Ciênc Farm Básica Apl. 2007;28(1):113-21.

2. Freitas $P$, Piccinato $C E$, Martins WP, Filho FM. Aterosclerose carotídea avaliada pelo eco-Doppler: associação com fatores de risco e doenças arteriais sistêmicas. J Vasc Bras. 2008;7(4):298-307. Disponível em: http://www.scielo.br/pdf/jvb/v7n4/0308.pdf

3. Locatelli EC, Pelizzari S, Scapini KB, Leguisamo $C P$, Silva $A B$. Exercícios físicos na doença arterial obstrutiva periférica. J Vasc Bras. 2009;8(3):00-0.

4. Marte AP, Santos RD. Bases fisiopatológicas da dislipidemia e hipertensão arterial. Rev Bras Hipertens. 2007;14(4):252-7.

5. Souza JEMR. A aterosclerose na visão do cardiologista: reconhecimento de uma doença sistêmica. Rev Bras Cardiol Invas. 2005;13(4):247.

6. Ferreira SRG, Moura EC, Malta DC, Sarno F. Freqüência de hipertensão arterial e fatores associados: Brasil, 2006. Rev Saúde Pública. 2009;43(Supl 2):98-106.

7. Tres GS, Utzig JB, Martins R, Heinrich S. Controle da pressão arterial, do diabetes mellitus e da dislipidemia na população de hipertensos de um

\section{CONCLUSÃO}

Atualmente, existe consenso entre os pesquisadores de que são necessárias mais pesquisas sobre as implicações dos fatores de risco para doenças cardiovasculares em idosos, devido a sua importância e impacto significativos, visando obter mais conhecimento delas e permitindo mudar o curso da doença e melhorar a qualidade de vida e aumentar a sobrevida de pacientes. A doença neste segmento da população, não é uma conseqüência inevitável do envelhecimento biológico, mas um processo patológico com fatores de risco que podem ser evitados.

Concluímos que a doença arterial do paciente é resultado da ação conjunta dos fatores de risco, hipertensão, diabetes mellitus, e da dislipidemia, segundo os critérios de Framingham.

ambulatório de residência médica. Rev Bras Hipertens. 2009;16(3):143-7.

8. Souza ARA, Costa A, Nakamura D, Mocheti LN, Filho PRS, Ovando LA. Um Estudo sobre hipertensão arterial sistêmica na cidade de Campo Grande, MS. Arq Bras Cardiol. 2007;88(4):441-6.

9. Carolino IDR, Fernandes CAM, Tasca RS, Marcon SS, Cuman RKN. Fatores de risco em pacientes com diabetes mellitus tipo II. Rev Latino-amer Enferm. 2008;16(2). Disponível em: http://www.scielo.br/pdf/ rlae/v16n2/pt_11.pdf

10. Bortolotto LA. Alterações das propriedades funcionais e estruturais de grandes artérias no diabetes mellitus. Arq Bras Endocrinol Metab. 2007;51/2. Disponível em: http://www.scielo.br/pdf/abem/v51n2/06.pdf.

11. Schutz R, Cantos GA, Cavalett C, Silva CSM, Hemes EM, Balen MG. Associações entre diferentes fatores de risco para doenças cardiovasculares e hipertensão arterial de pacientes com dislipidemia pertencentes a uma comunidade universitária. RBAC. 2008;40(2):979.

Artigo recebido em: 10/04/2011

Artigo aceito em: 12/07/2011 